\title{
Papers
}

\section{Use of a dummy (pacifier) during sleep and risk of sudden infant death syndrome (SIDS): population based case-control study}

De-Kun Li, Marian Willinger, Diana B Petitti, Roxana Odouli, Liyan Liu, Howard J Hoffman

\begin{abstract}
Objectives To examine the association between use of a dummy (pacifier) during sleep and the risk of sudden infant death syndrome (SIDS) in relation to other risk factors.

Design Population based case-control study.

Setting Eleven counties in California.

Participants Mothers or carers of 185 infants whose deaths were attributed to SIDS and 312 randomly selected controls matched for race or ethnicity and age.

Main outcome measure Use of a dummy during sleep determined through interviews.

Results The adjusted odds ratio for SIDS associated with using a dummy during the last sleep was 0.08 (95\% confidence interval 0.03 to 0.21 ). Use was associated with a reduction in risk in every category of sociodemographic characteristics and risk factors examined. The reduced risk associated with use seemed to be greater with adverse sleep conditions (such as sleeping prone or on side and sleeping with a mother who smoked), although the observed interactions were not significant. In addition, use of a dummy may reduce the impact of other risk factors for SIDS, especially those related to adverse sleep environment.For example, infants who did not use a dummy and slept prone or on their sides ( $v$ on their back) had an increased risk of SIDS (2.61, 1.56 to 4.38). In infants who used dummies, there was no increased risk associated with sleeping position $(0.66,0.12$ to 3.59$)$. While cosleeping with a mother who smoked was also associated with increased risk of SIDS among infants who did not use a dummy (4.5, 1.3 to 15.1), there was no such association among those who did (1.1, 0.1 to 13.4).

Conclusions Use of a dummy seems to reduce the risk of SIDS and possibly reduces the influence of known risk factors in the sleep environment.
\end{abstract}

\section{Introduction}

The reduction in the incidence of sudden infant death syndrome (SIDS) after the Back to Sleep campaign in the United States and other countries showed that sleep environment strongly influences the risk of SIDS, ${ }^{12}$ although the underlying mechanisms remain poorly understood. Despite the considerable reduction in the incidence of SIDS after this public health intervention, it remains one of the leading causes of infant mortality. Identification of new preventative measures to further reduce the incidence continues to be a priority. Some studies have reported that use of a dummy (pacifier) is associated with a reduced risk of SIDS, though few have examined the association in detail and in the context of other risk factors. ${ }^{3-7}$
Dummies usually have a bulky external handle, which could alter the infant's sleep environment by changing the configuration of the airway passage surrounding the nose and mouth. For example, pacifiers may prevent accidental hypoxia as a result of the face being buried into soft bedding or overlaying by objects (such as blankets, cosleepers, etc) by providing an air passage created by the bulky handle. Sucking on a dummy may enhance the development of neural pathways that control the potency of the upper airway. ${ }^{8}$

We examined data collected in a population based case-control study of risk factors for SIDS in California to determine whether use of a dummy during sleep is associated with a reduced risk of SIDS, what factors may modify the association between use and SIDS, and whether use influences other risk factors related to sleep environment.

\section{Methods}

We conducted a population based case-control study in 10 counties of northern California (Alameda, Contra Costa, Sacramento, San Francisco, Marin, San Mateo, Santa Clara, Monterey, San Joaquin, and Fresno) and Los Angeles County in southern California in 1997-2000. We identified cases of SIDS that were reported to the California Department of Health Services and to the coroner's office in Los Angeles County during the study period. In California, any cases with a diagnosis of SIDS or presumed SIDS have to be reported within 72 hours of diagnosis to the California SIDS Program, which in turn reports all cases to the state epidemiology and evaluation section at the department of health services. All cases must meet the diagnostic criteria of "the sudden unexpected death of an infant under 1 year of age, which remains unexplained after a postmortem examination (autopsy), death scene investigation, and review of the medical history."10 11 All cases with an initial diagnosis of presumed SIDS are given a final diagnosis of either SIDS or some other cause of death after further evaluation by coroners or medical examiners. We included in our analyses only cases with a final diagnosis of SIDS. Eligible participants were the mothers of any infants with a confirmed diagnosis of SIDS who lived in one of the participating counties and spoke either English or Spanish. A total of 185 mothers ( $50 \%$ of those eligible) participated in the study.

\section{Controls}

We used data from birth certificates to identify infants randomly selected from all eligible births in the same county in which the dead infant had lived, matched for maternal race/ethnicity (white, African-American, Hispanic, Asian, and other). Age at interview for controls was matched to age (plus or minus two 
weeks) at death for cases; and all mothers were asked about the index sleep, which for mothers of dead infants was defined as the last sleep and for mothers of control infants as the sleep during the night before the interview. These procedures were designed to improve comparability regarding the infant's developmental status and recall of the sleep environment of the infant. The mean age of the infants at death was 98 days and the mean age at interview for the controls was 104 days. This matching strategy was also meant to increase the comparability of sleeping environment between cases and controls, as sleeping behaviour and environment change with infant age. A total of 312 mothers of control infants ( $41 \%$ of those eligible) participated in the study.

Interviewers who were trained in grief counselling specifically related to SIDS obtained information on dummy use during the index sleep and on other environmental factors related to sleep, including sleep position. We also ascertained extensive information on risk factors for SIDS and potential confounders. Soft bedding was defined as heavy blankets, comforters, or sleeping bags used under the infant.

\section{Analysis}

We used odds ratios (95\% confidence intervals) to estimate the relative risk of SIDS associated with use of a dummy and unconditional logistic regression analysis to examine the relation between use during sleep and the risk after adjustment for potential confounders. All models included maternal race/ ethnicity, residence, and infant age (the matching variables). To examine the factors that may modify the relation between use of a dummy and the risk of SIDS, we stratified the analysis by potential modifying factors and compared the strength of the association across strata of the modifying factors.

\section{Results}

Table 1 shows the characteristics of cases and controls of our study population and the association between use of a dummy during the last sleep and the risk of SIDS (table 1). After adjustment for known confounders, the odds ratio for SIDS in infants who used a dummy during the last sleep was 0.08 (0.03 to 0.21 ), which translates to a more than $90 \%$ reduction of risk in this study compared with infants who did not use a dummy during the sleep.

To evaluate whether any factors might influence the association between use of a dummy and the reduced risk of SIDS, we examined the association separately according to factors related to the sleep environment as well as certain maternal and infant characteristics. All the factors examined have been directly or indirectly related to the risk of SIDS. We have reported unadjusted and adjusted odds ratios to enable comparison with previously reported odds ratios.

Use of a dummy during sleep was associated with a reduced the risk of SIDS in every category of those maternal and infant factors that we examined. In addition, the magnitude of the reduction was similar in the different levels of several maternal and infant characteristics examined, although the effect seemed to be stronger among infants of young mothers and married mothers, and first or second born infants (table 1).

There was a consistent trend towards use of a dummy during sleep being associated with a greater reduction of risk when an infant was in an adverse sleep environment, such as sleeping prone or on the side, sleeping with a mother who was a current smoker, or sleeping on soft bedding (table 1).

Infant thumb sucking itself was associated with a reduced risk of SIDS (adjusted odds ratio 0.43, 0.25 to 0.77 ). However, use of a dummy was associated with a reduced risk of SIDS regardless of whether the baby sucked its thumb $(0.07,0.01$ to 0.64 , with thumb sucking and $0.08,0.03$ to 0.23 , without) (table 1 ). In addition, use of a dummy was associated with a reduced risk in infants who ever breastfed and in infants currently breastfeeding $(0.09$, 0.03 to 0.25 ) (table 1 ).

To further evaluate whether use of a dummy could modify the effect of other risk factors related to the sleep environment, we examined the associations of sleep position, sleeping with a mother who smoked, and use of soft bedding with the risk in infants who did or did not use a dummy.

In infants who did not use a dummy during the last sleep, a prone or side sleeping position, sleeping with a mother who smoked, and sleeping on soft bedding during the last sleep were associated with increased risk. In infants who did use a dummy during the last sleep, however, these known risk factors no longer had an effect (table 2). None the less, the difference in odds ratios between infants who did or did not use a dummy was not significant (table 2).

\section{Discussion}

In this population based case-control study, use of a dummy was associated with a substantial reduction in the risk of SIDS. This reduced risk was independent of other known risk factors. Our observation confirms several previous reports. ${ }^{3-7}$ In addition, we examined the association of use of a dummy with the risk in relation to other risk factors, including those related to sleep environment. Under all conditions examined, use was associated with lower risk. The association of use of a dummy during sleep seemed stronger when an infant was already in an adverse sleep environment, such as in a prone or side sleep position, sleeping with a mother who smoked, or sleeping on soft bedding, although these differences did not reach significance. These preliminary findings need confirmation.

Our results also provide some evidence that use of a dummy may reduce the impact of other risk factors for SIDS, especially those related to adverse sleep conditions. The reduced impact did not reach significance in an interaction model. It is important that these findings be confirmed as they may provide new insight into the underlying mechanisms of the protective effect of dummies.

The use of dummies may be an effective strategy for public health intervention. While the rate of SIDS has declined in all populations in the United States, large racial disparities remain. In our study population, the incidence of use of dummies among populations at high risk of SIDS (African-Americans, young mothers, women with less than a college education, or low income) was comparable with that among those at lower risk of SIDS. Also, the reduced risk of SIDS associated with dummy use does not seem to vary by social and demographic characteristics. Therefore, advocating use of dummies for infants in high risk populations may have the potential to further reduce the incidence of SIDS.

\section{Limitations}

This was a case-control study, which is generally more susceptible to potential biases including selection and reporting biases. Results from case-control studies therefore require greater scrutiny and caution is needed in establishing a causal relation. Secondly, the sample sizes in some of the stratified analyses were small. The precision of the estimates is reflected in the width of corresponding 95\% confidence intervals. Thirdly, our discussion of potential underlying mechanisms of our observed association should not be confused with the "certainty" 
Table 1 Relation of dummy use with risk of SIDS by maternal and infant characteristics and sleep environment

SIDS cases

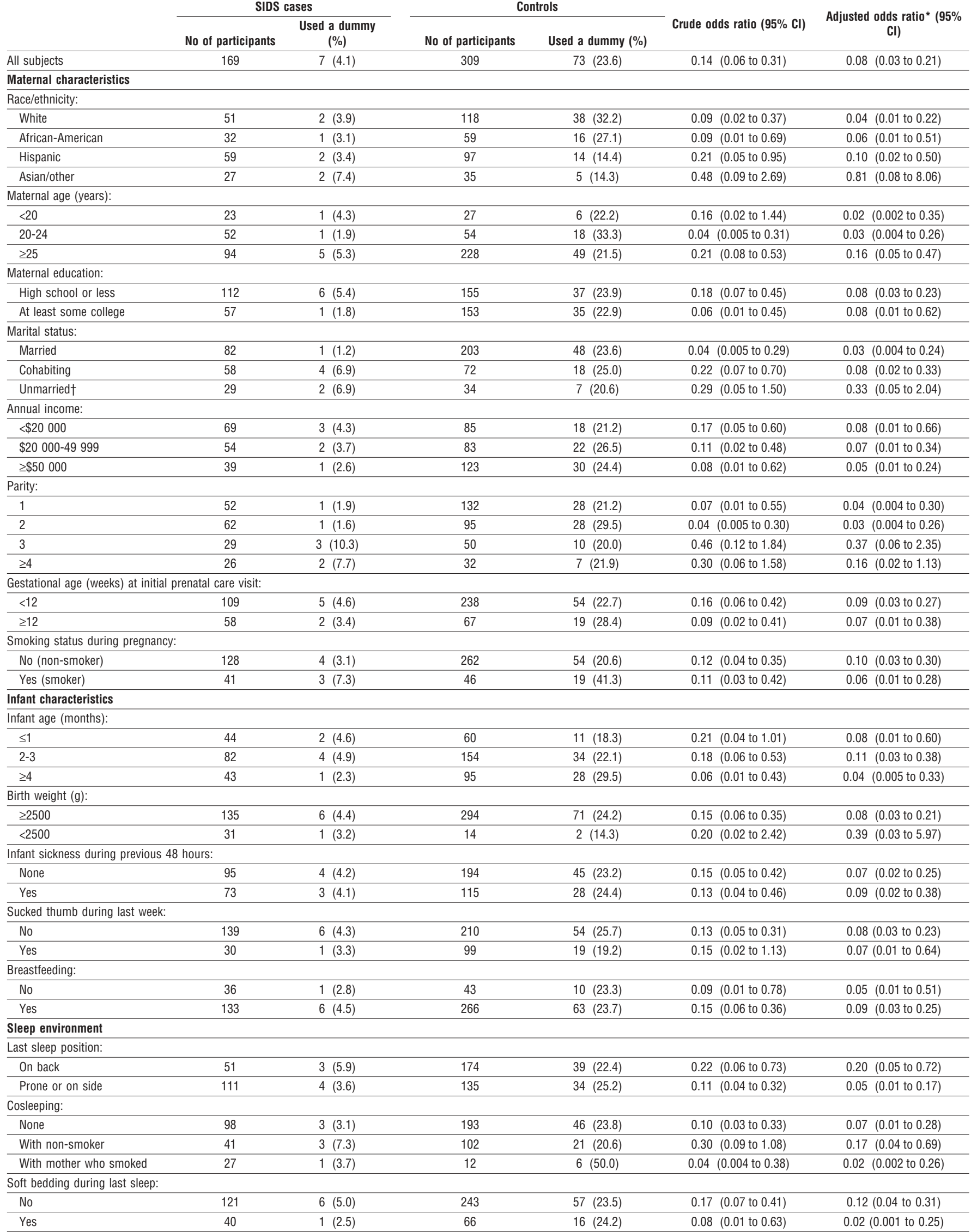

*Adjusted for maternal age, race, education, smoking during pregnancy, infant age, last sleep position, birth date, and region (study centre) unless variables themselves were examined as modifier.

†Includes unmarried/separated/divorced/widowed. 
Table 2 Dummy use in relation to selected risk factors for SIDS in sleep environment. Figures are numbers* (percentages) of infants with odds ratios

\begin{tabular}{|c|c|c|c|c|c|c|c|}
\hline & \multicolumn{3}{|c|}{ Did not use dummy } & \multicolumn{3}{|c|}{ Used a dummy } & \multirow{2}{*}{$\begin{array}{c}P \text { value for } \\
\text { difference in } O R\end{array}$} \\
\hline & Case $(n=162)$ & Control $(\mathrm{n}=236)$ & $\mathrm{OR} \dagger(95 \% \mathrm{CI})$ & Case $(n=7)$ & Control $(n=73)$ & OR† (95\% Cl) & \\
\hline \multicolumn{8}{|l|}{ Last sleep position: } \\
\hline On back $\ddagger$ & $48(31.0)$ & $135(57.2)$ & \multirow{2}{*}{$\begin{array}{c}2.61 \\
(1.56 \text { to } 4.38)\end{array}$} & $3(42.9)$ & $39(53.4)$ & \multirow{2}{*}{$\begin{array}{c}0.66 \\
(0.12 \text { to } 3.59)\end{array}$} & \multirow{2}{*}{0.38} \\
\hline Prone/on side & $107(69.0)$ & $101(42.8)$ & & $4(57.1)$ & $34(46.6)$ & & \\
\hline \multicolumn{8}{|l|}{ Cosleeping: } \\
\hline None/with non-smokerł & $133(83.6)$ & $228(97.4)$ & \multirow{2}{*}{$\begin{array}{c}4.50 \\
(1.32 \text { to } 15.10)\end{array}$} & $6(85.7)$ & $67(91.8)$ & \multirow{2}{*}{$\begin{array}{c}1.10 \\
(0.10 \text { to } 13.40)\end{array}$} & \multirow[b]{2}{*}{0.26} \\
\hline $\begin{array}{l}\text { With mother who } \\
\text { smoked }\end{array}$ & $26(16.4)$ & $6(2.6)$ & & $1(14.3)$ & $6(8.2)$ & & \\
\hline \multicolumn{8}{|l|}{ Soft bedding: } \\
\hline Noł & $115(74.7)$ & $186(78.8)$ & \multirow{2}{*}{$\begin{array}{c}1.42 \\
(0.79 \text { to } 2.57)\end{array}$} & $6(85.7)$ & $57(78.1)$ & \multirow{2}{*}{$\begin{array}{c}0.26 \\
(0.02 \text { to } 3.27)\end{array}$} & \multirow{2}{*}{0.15} \\
\hline Yes & $39(25.3)$ & $50(21.2)$ & & $1(14.3)$ & $16(21.9)$ & & \\
\hline
\end{tabular}

*Numbers in the table may not match the total numbers because of missing data.

†Adjusted for maternal age, race, education, and smoking during pregnancy, infant age and birth date, and region (study centre).

$\ddagger$ †eference group.

of a causal effect between use of dummies and risk of SIDS. The findings from our study and others will collectively provide support for the protective effect of dummies, but they are not "proof" of a causal effect by themselves.

In addition, the participation rate was low in our study. We examined the potential selection bias due to the low participation rate in a previous report from this study. ${ }^{1}$ We obtained birth certificates for all eligible infants, regardless of whether their mothers participated in the study, and calculated odds ratios of known risk factors for SIDS using information from the birth certificates. The odds ratios for infants under study were comparable with those obtained from all eligible infants, had they participated. ${ }^{1}$ Furthermore, the prevalence of use of dummies in our control group was comparable with that reported in other US populations. ${ }^{6}$ Finally, the magnitude of the odds ratio of SIDS associated with use of dummies was similar to that observed in other studies. ${ }^{6}$ While these observations reduce concerns of selection bias due to low participation, it remains a possibility.

Another possibility is that our results were biased by deliberate under-reporting by mothers of infants who died. However, we did not detect any deliberate under-reporting of several more well known risk factors, such as prone sleep position, maternal smoking, and cosleeping, which were more commonly reported among infants who died. It is unlikely that mothers would have chosen to deliberately under-report only use of dummies, which at the time of the interview was not generally known to the public as being related to sudden infant death in any way, while not under-reporting other well known risk factors.

\section{What is already known on this topic}

Sleep environment has consistently been reported as influencing the risk of sudden infant death syndrome (SIDS)

Use of a dummy during sleep has been reported as reducing the risk of SIDS

\section{What this study adds}

Use of a dummy during sleep was associated with a reduced risk of SIDS consistently across a wide range of socioeconomic characteristics and risk factor profiles

Use also reduced the adverse effects of a prone sleep position, sleeping with a mother who smoked, and soft bedding
Contributors: D-KL (guarantor) was responsible for the conception and design of the study, collection, analysis, and interpretation of the data, and preparation of the manuscript. MW and $\mathrm{HJH}$ contributed to the planning of the study and reviewed and revised drafts of the manuscript. DBP contributed to the planning of the study, oversaw its conduct at the Southern California site, and reviewed and made suggested revisions to early, intermediate, and final drafts of the manuscript. LL was involved in the analysis and interpretation of the data. RO was involved in the data collection and preparation of the manuscript.

Funding: National Institute of Child Health and Human Development (NICHD) and National Institute on Deafness and Other Communication Disorders (NIDCD), National Institutes of Health, contract N01-HD-53227.

Competing interest: None declared.

Ethical approval: Institutional Review Boards of Kaiser Permanente in Northern and Southern California and California State Committee for the Protection of Human Subjects.

1 Li DK, Petitti DB, Willinger M, McMahon R, Odouli R, Vu H, et al. Infant sleeping position and the risk of sudden infant death syndrome in California, 1997-2000. Am J Epidemiol 2003; 157:446-55

2 Willinger M, Ko CW, Hoffman HJ, Kessler RC, Corwin MJ. Factors associated with caregivers' choice of infant sleep position, 1994-1998: the national infant sleep position study. JAMA 2000;283:2135-42.

3 Arnestad M, Andersen M, Rognum TO. Is the use of dummy or carry-cot of importance for sudden infant death? Eur J Pediatr 1997;156:968-70.

4 Fleming PJ, Blair PS, Pollard K, Platt MW, Leach C, Smith I, et al. Pacifier use and sudden infant death syndrome: results from the CESDI/SUDI case control study. CESDI SUDI research team. Arch Dis Child 1999;81:112-6.

5 Mitchell EA, Taylor BJ, Ford RP, Stewart AW, Becroft DM, Thompson JM, et al. Dummies and the sudden infant death syndrome. Arch Dis Child 1993;68:501-4.

6 Hauck FR, Herman SM, Donovan M, Iyasu S, Merrick MC, Donoghue E, et al. Sleep environment and the risk of sudden infant death syndrome in an urban population: the Chicago infant mortality study. Pediatrics 2003;111:1207-14.

7 L'Hoir MP, Engelberts AC, van Well GT, Damste PH, Idema NK, Westers P, et al. Dummy use, thumb sucking, mouth breathing and cot death. Eur J Pediatr 1999;158:896-901

8 Franco P, Chabanski S, Scaillet S, Groswasser J, Kahn A. Pacifier use modifies infant's cardiac autonomic controls during sleep. Early Hum Dev 2004;77:99-108.

9 Franco P, Scaillet S, Wermenbol V, Valente F, Groswasser J, Kahn A. The influence of a pacifier on infants' arousals from sleep. J Pediatr 2000;136:775-9.

10 Zylke JW. Sudden infant death syndrome: resurgent research offers hope. JAMA 1989;262:1565-6.

11 Willinger M, James LS, Catz C. Defining the sudden infant death syndrome (SIDS): deliberations of an expert panel convened by the National Institute of Child Health and Human Development. Pediatr Pathol 1991;11:677-84.

(Accepted 8 November 2005)

doi $10.1136 /$ bmj.38671.640475.55

Division of Research, Kaiser Permanente Northern California, 2000 Broadway, Oakland, CA 94612

De-Kun Li senior research scientist

Liyan Liu programmer analyst

Roxana Odouli research associate

Research and Evaluation, Kaiser Permanente Southern California, Pasadena, CA 91188

Diana B Petitti senior scientific advisor

Pregnancy and Perinatology Branch, Centre for Research on Mothers and Children, National Institute of Child Health and Human Development, NIH, Bethesda, MD 20892

Marian Willinger health scientist administrator 
Epidemiology and Biostatistics Program, Division of Scientific Programs, National Institute on Deafness and Other Communication Disorders, NIH, Bethesda, MD 20892
Howard J Hoffman director

Correspondence to: Dr Li dkl@dor.kaiser.org 